\title{
INFINITESIMAL PERTURBATION ANALYSIS \\ FOR MAKE-TO-STOCK MANUFACTURING SYSTEMS BASED ON STOCHASTIC FLUID MODELS
}

\author{
Christos Panayiotou* Christos G. Cassandras **,1 \\ * Dept. of Electrical and Computer Engineering, University \\ of Cyprus. christosp@ucy.ac.cy \\ ** Dept. of Manufacturing Engineering, Boston University, \\ Brookline, MA 02446. cgc@bu.edu
}

\begin{abstract}
In this paper we study Make-To-Stock manufacturing systems and generalize the results obtained in Panayiotou et al. (2002). Specifically, we use the same modeling framework to derive sample derivatives of the objective function of interest with respect to buffer capacities (hedging points). In the earlier work, the input processes (machine processing and demand) were assumed piecewise constant. In this work, we generalize the results to piecewise differentiable processes. The derived estimates are unbiased and non-parametric, in the sense that they require no knowledge of the distributions of the underlying random processes. However, unlike the earlier results, these estimators require knowledge of some rates at certain points in time.
\end{abstract}

\section{INTRODUCTION}

This paper revisits the Make-To-Stock (MTS) problem (see Paschalidis et al. (2004), Panayiotou et al. (2002) and references therein). The objective is to determine the optimal capacities (or hedging points) of the finished goods and work-in-process buffers such that a cost function (to be defined) is minimized. Solving such problems usually relies on gradient information thus, estimating the gradient of the cost function with respect to these hedging points becomes an essential task.

To derive sensitivity information, we adopt a Stochastic Fluid Model (SFM), a framework commonly used to deal with the analysis and control of manufacturing systems, as in Suri and Fu (1991). We emphasize that our goal is to make

\footnotetext{
1 This work was supported in part by the National Science Foundation under Grants EEC-0088073 and DMI-0330171, by AFOSR under contract F49620-01-0056, and by ARO under grant DAAD19-01-0610.
}

use of such a model for the purpose of control and optimization rather than performance analysis; one can often identify the solution of an optimization problem based on such a model that captures only those features of the underlying "real" system that are needed to lead to the right solution. Such observations have been made in several contexts (e.g., Cassandras et al. (2002) and references therein). We derive the sensitivity information through Perturbation Analysis (PA) methods (Cassandras and Lafortune (1999)) by appropriately adapting them to a SFM viewed as a hybrid system.

In earlier work, we derived such sensitivity estimates when the input processes (machine processing and demand) were piecewise constant. Under this assumption, the analysis was done using finite differences. It was assumed that either of the hedging points was increased by $\Delta \theta$, and through geometric arguments, we derived the change in the buffer contents and the change in the per- 
formance measures of interest. In this work, we generalize those results to processes that are piecewise differentiable. The derived estimators still do not require any knowledge of the underlying stochastic processes, however, they require some rate information at certain points in time.

\section{MODEL}

In this paper we use the two machine model of Panayiotou et al. (2002) shown in Fig. 1. The upstream machine $M 2$ can process fluid at a maximum rate $\mu_{2}(t)$ and is assumed to have an infinite supply. The output of $M 2$ is placed in the work-in-process buffer $W$ which has capacity $\theta_{W}$. Machine $M 1$ drains $W$ at a maximum rate $\mu_{1}(t)$ and fills the finished part inventory buffer $F$. Finally, $F$ is drained by the demand at a rate $\rho(t)$. The random functions $\mu_{1}(t), \mu_{2}(t)$ and $\rho(t)$ are assumed piecewise differentiable w.p. 1. This is in contrast to Panayiotou et al. (2002) where they were assumed piecewise constant.

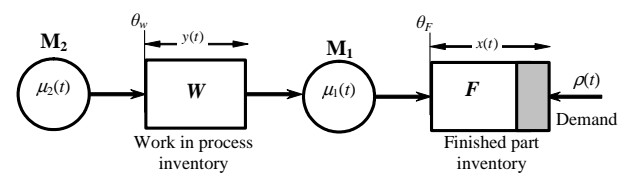

Fig. 1. System Model

Let $(x(t), y(t))$ denote the state of the system, where $x(t) \leq \theta_{F}, 0 \leq y(t) \leq \theta_{W}$ correspond to the amount of fluid present in $F$ and $W$ respectively. It is important to note that $x(t)$ can take negative values and these correspond to the amount of backlogged demand. Both, $x(t)$ and $y(t)$ depend on $\boldsymbol{\theta}$, however, to ease the notation, we omit $\boldsymbol{\theta}$. The dynamics of the system are given by

$$
\begin{aligned}
& \frac{d x(t)}{d t}=\left\{\begin{array}{cl}
\gamma_{1}(t ; \boldsymbol{\theta})-\rho(t) & \text { if } x(t)<\theta_{F}, \\
0 & \text { if } x(t)=\theta_{F}
\end{array}\right. \\
& \frac{d y(t)}{d t}=\left\{\begin{array}{cc}
\gamma_{2}(t ; \boldsymbol{\theta})-\gamma_{1}(t ; \boldsymbol{\theta}) \text { if } 0<y(t)<\theta_{W}, \\
0 & \text { if } y(t)=0 \text { or } y(t)=\theta_{W}
\end{array}\right.
\end{aligned}
$$

where $\gamma_{1}(t ; \boldsymbol{\theta})$ is the inflow rate to buffer $F$ and $\gamma_{2}(t ; \boldsymbol{\theta})$ is the inflow rate to buffer $W$. The rates $\gamma_{1}(t ; \boldsymbol{\theta})$ and $\gamma_{2}(t ; \boldsymbol{\theta})$ capture the coupling between the dynamics of the two buffers and are given by

$$
\begin{aligned}
& \gamma_{1}(t ; \boldsymbol{\theta})=\left\{\begin{array}{c}
\rho(t) \text { if } x(t)=\theta_{F} \\
\mu_{1}(t) \text { if } x(t)<\theta_{F} \text { and } y(t)>0, \\
\mu_{2}(t) \text { if } x(t)<\theta_{F} \text { and } y(t)=0
\end{array}\right. \\
& \gamma_{2}(t ; \boldsymbol{\theta})=\left\{\begin{array}{c}
\mu_{2}(t) \text { if } y(t)<\theta_{W} \\
\mu_{1}(t) \text { if } y(t)=\theta_{W} \text { and } x(t)<\theta_{F}(4) \\
\rho(t) \text { if } y(t)=\theta_{W} \text { and } x(t)=\theta_{F}
\end{array}\right.
\end{aligned}
$$

Fig. 2 shows a typical sample path for this system where we identify the following events. In general,

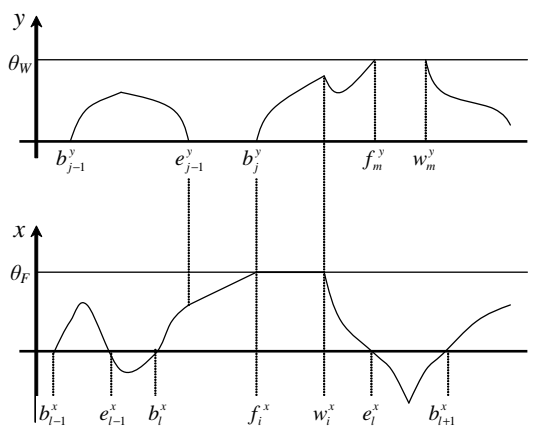

Fig. 2. Typical sample path

the time when these events occur depends on $\boldsymbol{\theta}$, however, to ease the notation, the explicit dependence is omitted.

$\beta_{i}^{q}$ : The trajectory of $q \in\{x, y\}$ ceases to be empty for the $i$ th time. This event occurs at time instants $b_{i}^{q}$.

$\epsilon_{i}^{q}$ : The trajectory of $q \in\{x, y\}$ becomes empty for the $i$ th time. It occurs at time instant $e_{i}^{q}$. $\phi_{i}^{q}$ : The trajectory of $q \in\{x, y\}$ becomes full for the $i$ th time. It occurs at time instant $f_{i}^{q}$.

$\omega_{i}^{q}$ : The trajectory of $q \in\{x, y\}$ ceases being full for the $i$ th time. It occurs at time instances $w_{i}^{q}$.

In the context of manufacturing systems of the type we study in this paper, one is typically interested in minimizing an expected cost function

$$
J_{T}(\boldsymbol{\theta})=\mathbb{E}\left[L_{T}(\boldsymbol{\theta})\right]
$$

where, $\boldsymbol{\theta}=\left[\theta_{F}, \theta_{W}\right]$ and $L_{T}(\boldsymbol{\theta})$ is a sample function which in general has the form

$L_{T}(\boldsymbol{\theta})=\frac{1}{T} \int_{0}^{T}\left(c_{F}[x(t)]^{+}+c_{W} y(t)+c_{D}[-x(t)]^{+}\right) d t$

The first two terms correspond to the average inventory cost in $F$ and $W$ respectively and the third one corresponds to the average cost of backlogged demand. Furthermore, $[x]^{+}=\max \{0, x\}$ and $c_{F}, c_{W}, c_{D}$ are non-negative constants. We are interested in obtaining estimators of $d J_{T} / d \theta$ based on observed data which are then used in stochastic approximation algorithms as in Panayiotou et al. (2002) and Paschalidis et al. (2004).

\section{INFINITESIMAL PERTURBATION ANALYSIS (IPA)}

In this section we derive sample derivatives for each of the components that make up the sample function $L_{T}(\boldsymbol{\theta}), \boldsymbol{\theta}=\left[\theta_{F}, \theta_{W}\right]$. So we define

$$
\begin{gathered}
X_{T}(\boldsymbol{\theta})=\int_{0}^{T} x(t) d t, Y_{T}(\boldsymbol{\theta})=\int_{0}^{T} y(t) d t \\
X_{T}^{+}(\boldsymbol{\theta})=\int_{0}^{T}[x(t)]^{+} d t, X_{T}^{-}(\boldsymbol{\theta})=\int_{0}^{T}[-x(t)]^{+} d t(6)
\end{gathered}
$$


In our earlier approach (Panayiotou et al. (2002)), we derived such IPA estimates under the assumption of piecewise constant input processes $\left(\mu_{1}(t)\right.$, $\mu_{2}(t)$, and $\left.\rho(t)\right)$. In that work, we assumed that $\theta_{q}$ is increased by $\Delta \theta_{q}>0, q \in\{F, W\}$ and determined the change in $X_{T}$ and $Y_{T}, \Delta X_{T}$ and $\Delta Y_{T}$ respectively. In this paper, we generalize the results for piecewise differentiable input processes. The derivation of the IPA derivative estimates is by differentiating the sample functions $X_{T}(\cdot)$, and $Y_{T}(\cdot)$ directly. First, we make the following assumptions and derive some preliminary results.

\section{Assumptions:}

A1: W.p. 1, the functions $\mu_{1}(t), \mu_{2}(t)$ and $\rho(t)$ are piecewise analytic in the interval $[0, T]$.

A2: W.p. 1, except of $\left(\epsilon^{y}, \omega^{x}\right),\left(\omega^{x}, \omega^{y}\right)$ and $\left(\phi^{x}, \beta^{y}\right)$ no two events can occur at the same time.

A3: W.p. 1, there does not exist an interval $(s, s+$ $\tau), \tau>0$, such that $M_{n, m}(t)=0$, where

$$
M_{n, m}(t)=\mu_{n}(t)-\mu_{m}(t)
$$

$n \neq m, n, m \in\{0,1,2\}$ and, by definition, $\mu_{0}(t)=\rho(t)$.

In A2, the exceptions are needed since in each pair, it is possible that the occurrence of the first event might also trigger the second event. All three assumptions are mild and are satisfied for general systems. A3 is rather technical. If it is violated, then one can still resort to one sided derivatives and get similar results, but we keep it to simplify our analysis. A1-A3 guarantee the existence of the sample derivatives $\frac{d L_{T}}{d \theta_{F}}$ and $\frac{d L_{T}}{d \theta_{W}}$.

\subsection{Preliminary Results}

In this section we are concerned with the evaluation of event time derivatives (with respect to either $\theta_{F}$ of $\theta_{W}$ ) of some critical events. We derive a number of iterative relations for evaluating the required event time derivatives, but first, we introduce the following sample path decomposition.

3.1.1. Sample Path Decomposition In this section we decompose the sample paths of $(x(t), y(t))$ into boundary periods (BP) and non-boundary periods (NBP). BPs, are intervals where the buffer content is at some boundary, i.e., $x(t)=\theta_{F}$ and $y(t)=0$ or $y(t)=\theta_{W}$. A NBP followed by a $\mathrm{BP}$ form a cycle. We also define the following variables. Let $v_{i, 0}$ denote the beginning point of the $i$ th cycle in the sample path of $x(t)$, therefore, it also denotes the beginning of the $i$ th NBP of $x(t)$. This point coincides with the occurrence of the $\omega_{i-1}^{x}$ event (buffer $F$ ceases to be full). $v_{i, 1}$ denotes the end of the $i$ th NBP (and the beginning of the $i$ th $\mathrm{BP}$ ) while $v_{i, 2}$ denotes the end point of the $i$ th BP. $v_{i, 1}$ and $v_{i, 2}$ coincide with the $\phi_{i}^{x}$ and $\omega_{i}^{x}$ events respectively. Similarly, for the $y(t)$ sample path, let $s_{i, 0}$ denote the beginning of the $i$ th NBP, $s_{i, 1}$ the end of the $i$ th NBP (same as the start of the $i$ th $\mathrm{BP}$ ) and $s_{i, 2}$ the end of the $i$ th BP. Points $s_{i, 0}$ and $s_{i, 2}$ occur due to either $\beta^{y}$ or $\omega^{y}$ events ( $W$ ceases to be empty or full respectively). Point $s_{i, 1}$ occurs due to either $\phi^{y}$ or $\epsilon^{y}$ events ( $W$ becomes full or empty respectively). Furthermore, we use the notation $\mathcal{C}_{i}^{x}=\left[v_{i, 0}, v_{i, 2}\right)$, $i=1, \cdots, N_{T}^{x}, \mathcal{C}_{j}^{y}=\left[s_{j, 0}, s_{j, 2}\right), j=1, \cdots, N_{T}^{y}$, to denote the cycles in the sample paths of $x(t)$ and $y(t)$ respectively; $N_{T}^{x}, N_{T}^{y}$ denote the random number of cycles in the corresponding sample path during the observation interval $[0, T]$.

\subsubsection{Event Time Derivatives}

Lemma 1. Let $w_{i}^{x}$ denote the time when the $\omega_{i}^{x}$ event ( $\mathrm{F}$ ceases to be full) has occurred. Then,

$$
\begin{aligned}
& \frac{\partial w_{i}^{x}}{\partial \theta_{F}}=\left\{\begin{array}{cc}
\frac{\partial e_{j}^{y}}{\partial \theta_{F}} & \text { if } w_{i}^{x}=e_{j}^{y} \\
0 & \text { otherwise }
\end{array}\right. \text { and } \\
& \frac{\partial w_{i}^{x}}{\partial \theta_{W}}=\left\{\begin{array}{cc}
\frac{\partial e_{j}^{y}}{\partial \theta_{W}} & \text { if } w_{i}^{x}=e_{j}^{y}, \\
0 & \text { otherwise }
\end{array}\right.
\end{aligned}
$$

for all $i=1,2, \cdots$ and some $j=1,2, \cdots{ }^{2}$.

Lemma 2. Let $w_{i}^{y}$ denote the time when the $\omega_{i}^{y}$ event (W ceases to be full) has occurred. Then ${ }^{3}$,

$$
\frac{\partial w_{i}^{y}}{\partial \theta_{F}}=\frac{\partial w_{i}^{y}}{\partial \theta_{W}}=0, \text { for all } i=1,2, \cdots .
$$

Lemma 3. Let $b_{i}^{y}$ denote the time when the $i$ th $\beta^{y}$ event (W ceases to be empty) has occurred. Then,

$$
\begin{aligned}
& \frac{\partial b_{i}^{y}}{\partial \theta_{F}}=\left\{\begin{array}{cc}
\frac{\partial f_{j}^{x}}{\partial \theta_{F}} & \text { if } b_{i}^{y}=f_{j}^{x} \\
0 & \text { otherwise }
\end{array}\right. \text { and } \\
& \frac{\partial b_{i}^{y}}{\partial \theta_{W}}=\left\{\begin{array}{cc}
\frac{\partial f_{j}^{x}}{\partial \theta_{W}} & \text { if } b_{i}^{y}=f_{j}^{x}, \\
0 & \text { otherwise }
\end{array}\right.
\end{aligned}
$$

for all $i=1,2, \cdots$ and some $j=1,2, \cdots{ }^{4}$. Also, $f_{j}^{y}=v_{j, 1}, j=1,2, \cdots$.

Lemmas 1 and 3 show the coupling between the two sample paths. Next we derive four lemmas that show how perturbations in event times propagate from the beginning of the NBP to the end

\footnotetext{
2 Using the decomposition of Sec. 3.1.1, $w_{i}^{x}=v_{i, 2}=$ $v_{i+1,0}$ for all $i=1,2, \cdots$ and $e_{j}^{y}=s_{n, 1}, n \geq j=1,2, \cdots$ ( $n$ also includes all NBPs that end with a $\phi^{y}$ event).

$3 w_{i}^{y}=s_{n, 2}=s_{n+1,0}$ for all $n \geq i(n$ includes all BPs that end with a $\beta^{y}$ event).

${ }^{4} b_{i}=s_{n, 2}=s_{n+1,0}$ where again $n \geq i$ since it may include NBPs that start with an event $\omega^{y}$.
} 
of the same NBP. The first two assume that no events occur during the NBP while the remaining two assume an arbitrary number of events. First, for notational economy we define the following indicator functions.

$$
\mathbf{1}_{F}[t]=\mathbf{1}\left[x(t)=\theta_{F}\right], \mathbf{1}_{W}[t]=\mathbf{1}\left[y(t)=\theta_{W}\right] .
$$

Lemma 4. Let the interval $\left[s_{i, 0}, s_{i, 1}\right) \in\left[v_{j, 0}, v_{j, 1}\right)$ or $\left[s_{i, 0}, s_{i, 1}\right) \in\left[v_{j, 1}, v_{j, 2}\right)$, for some $i, j=1,2, \cdots$, then

$$
\begin{aligned}
\frac{\partial s_{i, 1}}{\partial \theta_{F}} & =\frac{M_{2, g}\left(s_{i, 0}\right)}{M_{2, g}\left(s_{i, 1}\right)} \frac{\partial s_{i, 0}}{\partial \theta_{F}} \\
\frac{\partial s_{i, 1}}{\partial \theta_{W}} & =\frac{\mathbf{1}_{W}\left[s_{i, 1}\right]-\mathbf{1}_{W}\left[s_{i, 0}\right]}{M_{2, g}\left(s_{i, 1}\right)}+\frac{M_{2, g}\left(s_{i, 0}\right)}{M_{2, g}\left(s_{i, 1}\right)} \frac{\partial s_{i, 0}}{\partial \theta_{W}}
\end{aligned}
$$

where $M_{n, m}(t)$ is defined in $(7)$ and $g=\mathbf{1}\left[x(t)<\theta_{F}\right]$, $t \in\left[s_{i, 0}, s_{i, 1}\right)$.

Lemma 5. Let the interval $\left[v_{i, 0}, v_{i, 1}\right) \in\left[s_{j, 0}, s_{j, 1}\right)$ or $\left[v_{i, 0}, v_{i, 1}\right) \in\left[s_{j, 1}, s_{j, 2}\right)$, for some $i, j=1,2, \cdots$, then

$$
\begin{aligned}
& \frac{\partial v_{i, 1}}{\partial \theta_{F}}=-\frac{1-\mathbf{1}_{F}\left[v_{i, 0}\right]}{M_{g, 0}\left(v_{i, 1}\right)}+\frac{M_{g, 0}\left(v_{i, 0}\right)}{M_{g, 0}\left(v_{i, 1}\right)} \frac{\partial v_{i, 0}}{\partial \theta_{F}} \\
& \frac{\partial v_{i, 1}}{\partial \theta_{W}}=\frac{M_{g, 0}\left(v_{i, 0}\right)}{M_{g, 0}\left(v_{i, 1}\right)} \frac{\partial v_{i, 0}}{\partial \theta_{W}}
\end{aligned}
$$

where $M_{n, m}(t)$ is defined in (7) and $g=1+$ $\mathbf{1}[y(t)=0], t \in\left[v_{i, 0}, v_{i, 1}\right)$.

Lemmas 4 and 5 provide an iterative algorithm for determining the event time derivatives (with respect to either $\theta_{F}$ of $\theta_{W}$ ) of the end point of a NBP given the event time derivatives of the starting point of a NBP, when no other event occurs during the period. Finally, we need to determine how a perturbation propagates from the beginning of a NBP to the end of a NBP when several other events occur during the interval. However, we first need to define the following notation. We concentrate in an interval $\left[v_{i, 0}, v_{i, 1}\right)$ and identify a sequence of points $\xi_{i, j}, j=0, \cdots, K_{i}^{y}+1$ where $\xi_{i, 0}=v_{i, 0}, \xi_{i, K_{i}^{y}+1}=v_{i, 1}$ and at any other point $\xi_{i, j}, y\left(\xi_{i, j}\right)$ transitions either from empty to nonempty or vice versa. In other words, $\xi_{i, j}$ is a sequence with all $\beta^{y}$ or $\epsilon^{y}$ events in the interval $\left[v_{i, 0}, v_{i, 1}\right)$. Next, we also define the index function that takes values in $\{1,2\}$

$$
\begin{aligned}
g_{i}(k) & =\left\{\begin{array}{l}
1 \text { if } g_{i}(k-1)=2 \\
2 \text { if } g_{i}(k-1)=1
\end{array}\right. \\
\text { with } g_{i}(0) & =\left\{\begin{array}{l}
1 \text { if } y\left(\xi_{i, 0}\right)>0 \\
2 \text { if } y\left(\xi_{i, 0}\right)=0
\end{array}\right.
\end{aligned}
$$

Lemma 6. The derivatives of $v_{i, 1}, i=1,2, \cdots$ with respect to $\theta_{F}$ and $\theta_{W}$ are given by

$$
\begin{aligned}
v_{i, 1}^{\prime} & =-\left(M_{g_{i}\left(K_{i}^{y}\right), 0}\left(v_{i, 1}\right)\right)^{-1} \times \\
& \left(-\rho\left(v_{i, 0}\right) v_{i, 0}^{\prime}+\sum_{j=1}^{K_{i}^{y}} M_{g_{i}(j-1), g_{i}(j)}\left(\xi_{i, j}\right) \xi_{i, j}^{\prime}\right)
\end{aligned}
$$

where the prime notation indicates the event time derivatives with respect to either $\theta_{F}$ or $\theta_{W}$.

As mentioned above, all $\xi_{i, j} j=1, \cdots, K_{i}^{y}$ event times correspond to events in the $y(t)$ sample path. These either end a NBP (e.g., $\epsilon^{y}$ events) or start a new NBP (e.g., $\beta^{y}$ events). For the first, we use either Lemma 4 or 7 below, to determine the event time derivative given the event time derivative of the start of the NBP. For the second, we use Lemmas 3 and 2 to determine the event time derivative of the NBP starting point.

Next, we analyze the interval $\left[s_{i, 0}, s_{i, 1}\right) i=$ $1,2, \cdots$, and we identify a sequence of points $\zeta_{i, j}$, $j=0, \cdots, K_{i}^{x}+1$ where $\zeta_{i, 0}=s_{i, 0}, \zeta_{K_{i}^{x}+1}=s_{i, 1}$ and at any other point $x\left(\zeta_{i, j}\right)$ transitions either from full to non-full or vice versa. In other words, $\zeta_{i, j}$ is a sequence with all $\phi^{x}$ or $\omega^{x}$ events in the interval $\left[s_{i, 0}, s_{i, 1}\right)$. Next, we also define the index function that takes values in $\{1,2\}$

$$
\begin{aligned}
h_{i}(k) & =\left\{\begin{array}{l}
0 \text { if } h_{i}(k-1)=1 \\
1 \text { if } h_{i}(k-1)=0
\end{array}\right. \\
\text { with } h_{i}(0) & =\left\{\begin{array}{l}
0 \text { if } x\left(\zeta_{i, 0}\right)=\theta_{F} \\
1 \text { if } x\left(\zeta_{i, 0}\right)<\theta_{F}
\end{array}\right.
\end{aligned}
$$

Lemma 7. The derivatives of $s_{i, 1}, i=1,2, \cdots$ with respect to $\theta_{F}$ are given by

$$
\begin{aligned}
s_{i, 1}^{\prime} & =-\left(M_{2, h_{i}\left(K_{i}^{x}\right)}\left(s_{i, 1}\right)\right)^{-1} \times \\
& \left(\mu_{2}\left(s_{i, 0}\right) s_{i, 0}^{\prime}+\sum_{j=1}^{K_{i}^{x}} M_{h_{i}(j-1), h_{i}(j)}\left(\zeta_{i, j}\right) \zeta_{i, j}^{\prime}\right) .
\end{aligned}
$$

The derivatives of $s_{i, 1}, i=1,2, \cdots$ with respect to $\theta_{W}$ are given by

$$
\begin{aligned}
& s_{i, 1}^{\prime}=-\left(M_{2, h_{i}\left(K_{i}^{x}\right)}\left(s_{i, 1}\right)\right)^{-1} \times \\
& \left(\mathbf{1}_{W}\left[s_{i, 1}\right]-\mathbf{1}_{W}\left[s_{i, 0}\right]+\mu_{2}\left(s_{i, 0}\right) s_{i, 0}^{\prime}\right. \\
& \left.+\sum_{j=1}^{K_{i}^{y}} M_{h_{i}(j-1), h_{i}(j)}\left(\zeta_{i, j}\right) \zeta_{i, j}^{\prime}\right)
\end{aligned}
$$

Note that all $\zeta_{i, j} j=1, \cdots, K_{i}^{y}$ times correspond to events in the $x(t)$ sample path. These fall into two categories. (i) End points of BPs (or beginning points of NBPs) i.e., events $\omega^{x}$. Therefore, the corresponding event time derivatives are determined by Lemmas 1. (ii) End points of NBPs of $x(t)$. In this case, use Lemma 5 or 6 . 


\subsection{Sample Function Derivatives}

Now we are ready to derive the derivatives of the sample functions of interest with respect to either $\theta_{F}$ or $\theta_{W}$. First, using cycles, observe that $X_{T}(\boldsymbol{\theta})$ and $Y_{T}(\boldsymbol{\theta})$ can be written as

$$
\begin{aligned}
& X_{T}(\boldsymbol{\theta})=\sum_{i=1}^{N_{T}^{x}}\left(\int_{v_{i, 0}}^{v_{i, 1}} x(t) d t+\theta_{F}\left(v_{i, 2}-v_{i, 1}\right)\right) \\
& Y_{T}(\boldsymbol{\theta})=\sum_{i=1}^{N_{T}^{y}}\left(\int_{s_{i, 0}}^{s_{i, 1}} y(t) d t+\theta_{W}\left(s_{i, 2}-s_{i, 1}\right) \mathbf{1}_{W}\left[s_{i, 1}\right]\right)
\end{aligned}
$$
3.2.1. Evaluation of $\frac{\partial X_{T}(\boldsymbol{\theta})}{\partial \theta_{F}}$
with respect to $\theta_{F}$ we get

Differentiating (11)

$$
\frac{\partial X_{T}(\boldsymbol{\theta})}{\partial \theta_{F}}=\sum_{i=1}^{N_{T}^{x}}\left(\left(v_{i, 2}-v_{i, 1}\right)+\int_{v_{i, 0}}^{v_{i, 1}} x^{\prime}(t) d t\right)
$$

where we use the prime notation to denote the derivative of a quantity with respect to $\theta_{F}$. To obtain this result we used the facts that $v_{i, 2}=$ $v_{i+1,0}, i=0, \cdots, N_{T}^{x}-1, v_{N_{T}^{x}, 2}^{\prime}=T^{\prime}=0$ and $v_{1,0}^{\prime}=0^{\prime}=0$. During the $i$ th $\mathrm{NBP}^{5}$

$$
x(t)=\theta_{F} \mathbf{1}_{F}\left[v_{i, 0}\right]+\int_{v_{i, 0}}^{t}\left(\gamma_{1}(\tau ; \boldsymbol{\theta})-\rho(\tau)\right) d \tau .
$$

Again, we identify a sequence of points $\xi_{i, j}, j=$ $0, \cdots, K_{i}^{y}+1$ where $\xi_{i, 0}=v_{i, 0}, \xi_{i, K_{i}^{y}+1}=v_{i, 1}$ and at any other point $\xi_{i, j}, y\left(\xi_{i, j}\right)$ transitions either from empty to non-empty or vice versa. Using this segmentation, we can rewrite $x(t)$

$$
x(t)=\theta_{F} \mathbf{1}_{F}\left[v_{i, 0}\right]+\sum_{j=0}^{K_{i}^{y}(t)} \int_{\xi_{i, j}}^{\xi_{i, j+1}} M_{g_{i}(j), 0}(\tau) d \tau .
$$

where $K_{i}^{y}(t)$ is the number of $\epsilon^{y}$ and $\beta^{y}$ events in $\left[v_{i, 0}, t\right)$ and $\xi_{K_{i}^{y}(t)+1}=t\left(g_{i}(j)\right.$ is given by $(9)$ and $M_{i, j}(\cdot)$ by $\left.(7)\right)$. Next, we differentiate $x(t)$ with respect to $\theta_{F}$ :

$$
\begin{aligned}
& x^{\prime}(t)=\mathbf{1}_{F}\left[v_{i, 0}\right]+\rho\left(\xi_{i, 0}\right) \xi_{i, 0}^{\prime}+ \\
& \quad \sum_{j=0}^{K_{i}^{y}(t)}\left[\mu_{g_{i}(j)}\left(\xi_{i, j+1}\right) \xi_{i, j+1}^{\prime}-\mu_{g_{i}(j)}\left(\xi_{i, j}\right) \xi_{i, j}^{\prime}\right](16)
\end{aligned}
$$

where as earlier, $\xi_{i, j}^{\prime}$ indicates the derivative of the time instant $\xi_{i, j}$ with respect to $\theta_{F}$. Also, $\xi_{i, K_{i}^{y}(t)+1}^{\prime}=t^{\prime}=0$. This is a piecewise constant function that changes value at points $t=\xi_{i, j}$, $j=0, \cdots, K_{i}^{y}$ as more terms are added in the sum. The event time derivatives with respect to $\theta_{F}$ are determined by Lemmas 2, 3, 4 and 7 .

\footnotetext{
$5 \mathbf{1}_{F[}[\cdot]$ is needed since $x(0)=0$.
}

Theorem 1. The derivative of $X_{T}(\boldsymbol{\theta})$ with respect to $\theta_{F}$ is given by

$$
\begin{aligned}
\frac{\partial X_{T}(\boldsymbol{\theta})}{\partial \theta_{F}}= & \sum_{i=1}^{N_{T}^{x}}\left(v_{i, 2}-v_{i, 1}\right) \\
& +\sum_{i=1}^{N_{T}^{x}} \sum_{j=1}^{K_{i}^{y}+1} x^{\prime}\left(\xi_{i, j}\right)\left(\xi_{i, j}-v_{i, 0}\right)
\end{aligned}
$$

where $x^{\prime}\left(\xi_{i, j}\right), j=1, \cdots, K_{i}^{y}+1$ is the derivative of $x(t)$ with respect to $\theta_{F}$ and is evaluated by (16) for all $i=1, \cdots, N_{T}^{x}$.

3.2.2. Evaluation of $\frac{\partial X_{T}(\boldsymbol{\theta})}{\partial \theta_{W}}$

Differentiating (11) with respect to $\theta_{W}$ we get

$$
\frac{\partial X_{T}(\boldsymbol{\theta})}{\partial \theta_{W}}=\sum_{i=1}^{N_{T}^{x}} \int_{v_{i, 0}}^{v_{i, 1}} x^{\prime}(t) d t
$$

where now the prime notation denotes the derivative of a quantity with respect to $\theta_{W}$. Next, we evaluate $x^{\prime}(t)=\frac{\partial x(t)}{\partial \theta_{W}}$. During the $i$ th NBP, $x(t)$ is given by (15) where the same segmentation as in the previous case still holds. Next, we differentiate $x(t)$ with respect to $\theta_{W}$ to obtain

$$
\begin{aligned}
& x^{\prime}(t)=\rho\left(\xi_{i, 0}\right) \xi_{i, 0}^{\prime} \\
& \quad+\sum_{j=0}^{K_{i}^{y}(t)}\left[\mu_{g_{i}(j)}\left(\xi_{i, j+1}\right) \xi_{i, j+1}^{\prime}-\mu_{g_{i}(j)}\left(\xi_{i, j}\right) \xi_{i, j}^{\prime}(18)\right.
\end{aligned}
$$

where now, $\xi_{i, j}^{\prime}$ is the derivative of $\xi_{i, j}$ with respect to $\theta_{W}$. This is again a piecewise constant function. The event time derivatives are again determined by Lemmas 2, 3, 4 and 7 .

Theorem 2. The derivative of $X_{T}(\boldsymbol{\theta})$ with respect to $\theta_{W}$ is given by

$$
\frac{\partial X_{T}(\boldsymbol{\theta})}{\partial \theta_{W}}=\sum_{i=1}^{N_{T}^{x}} \sum_{j=1}^{K_{i}^{y}+1} x^{\prime}\left(\xi_{i, j}\right)\left(\xi_{i, j}-v_{i, 0}\right)
$$

where $x^{\prime}\left(\xi_{i, j}\right), j=1, \cdots, K_{i}^{y}+1$ is the derivative of $x(t)$ with respect to $\theta_{W}$ and is evaluated by (18) for all $i=1, \cdots, N_{T}^{x}$.

3.2.3. Evaluation of $\frac{\partial Y_{T}(\boldsymbol{\theta})}{\partial \theta_{F}}$

Differentiating (12) with respect to $\theta_{F}$ we get

$$
\frac{\partial Y_{T}(\boldsymbol{\theta})}{\partial \theta_{F}}=\sum_{i=1}^{N_{T}^{y}} \int_{s_{i, 0}}^{s_{i, 1}} y^{\prime}(t) d t
$$

where we use the prime notation to denote the derivative of a quantity with respect to $\theta_{F}$. Also, we used the fact that $s_{i, 2}=s_{i+1,0}$. Next, we evaluate $y^{\prime}(t)=\frac{\partial y(t)}{\partial \theta_{F}}$. During the $i$ th NBP, 


$$
y(t)=\theta_{W} \mathbf{1}_{W}\left[s_{0}\right]+\int_{v_{i, 0}}^{t}\left(\mu_{2}(\tau)-\gamma_{1}(\tau ; \boldsymbol{\theta})\right) d \tau .
$$

As in previous cases, in the interval $\left[s_{i, 0}, s_{i, 1}\right)$ $i=1,2, \cdots$ we identify a sequence of points $\zeta_{i, j}$, $j=0, \cdots, K_{i}^{x}+1$ where $\zeta_{i, 0}=s_{i, 0}, \zeta_{K_{i}^{x}+1}=s_{i, 1}$ and at any other point $x\left(\zeta_{i, j}\right)$ transitions either from full to non-full or vice versa. Using this segmentation we rewrite $y(t)$

$$
y(t)=\theta_{W} \mathbf{1}_{W}\left[s_{0}\right]+\sum_{j=0}^{K_{i}^{x}(t)} \int_{\zeta_{i, j}}^{\zeta_{i, j+1}} M_{2, h_{i}(j)}(\tau) d \tau .
$$

where $K_{i}^{x}(t)$ is the number of $\phi^{x}$ and $\omega^{x}$ events in $\left[s_{i, 0}, t\right)$. By convention we set $\rho(t)=\mu_{0}(t)$ and $h_{i}(j)$ is given by (10). Next, we differentiate $y(t)$ with respect to $\theta_{F}$ to obtain

$$
\begin{aligned}
& y^{\prime}(t)=-\mu_{2}\left(\zeta_{i, 0}\right) \zeta_{i, 0}^{\prime} \\
& \quad+\sum_{j=0}^{K_{i}^{x}(t)}\left[\mu_{h_{i}(j)}\left(\zeta_{i, j+1}\right) \zeta_{i, j+1}^{\prime}-\mu_{h_{i}(j)}\left(\zeta_{i, j}\right) \zeta_{i, j}^{\prime}\right](22)
\end{aligned}
$$

where as earlier, $\zeta_{i, j}^{\prime}$ is the derivative of $\zeta_{i, j}$ with respect to $\theta_{F}$. This is also a piecewise constant function. The event time derivatives with respect to $\theta_{F}$ are determined by the Lemmas 1,5 and 6 .

Theorem 3. The derivative of $Y_{T}(\boldsymbol{\theta})$ with respect to $\theta_{F}$ is given by

$$
\frac{\partial Y_{T}(\boldsymbol{\theta})}{\partial \theta_{F}}=\sum_{i=1}^{N_{T}^{y}} \sum_{j=1}^{K_{i}^{x}+1} y^{\prime}\left(\zeta_{i, j}\right)\left(\zeta_{i, j}-s_{i, 0}\right)
$$

where $y^{\prime}\left(\zeta_{i, j}\right), j=1, \cdots, K_{i}^{x}+1$ is the derivative of $y(t)$ with respect to $\theta_{F}$ and is evaluated by (22) for all $i=1, \cdots, N_{T}^{y}$.

\subsubsection{Evaluation of $\frac{\partial Y_{T}(\boldsymbol{\theta})}{\partial \theta_{W}}$}

Differentiating (12) with respect to $\theta_{W}$ we get

$$
\frac{\partial Y_{T}(\boldsymbol{\theta})}{\partial \theta_{W}}=\sum_{i=1}^{N_{T}^{y}}\left(\left(s_{i, 2}-s_{i, 1}\right) \mathbf{1}_{W}\left[s_{i, 1}\right]+\int_{s_{i, 0}}^{s_{i, 1}} y^{\prime}(t) d t\right)
$$

where now, the prime notation denotes the derivative of a quantity with respect to $\theta_{W}$. Also, in the last step we used the fact that $s_{i, 2}=s_{i+1,0}$. Next, we evaluate $y^{\prime}(t)=\frac{\partial y(t)}{\partial \theta_{W}}$. During the $i$ th NBP, $y(t)$ is given by $(21)$ and we differentiate $y(t)$ with respect to $\theta_{W}$ to obtain

$$
\begin{aligned}
& y^{\prime}(t)=\mathbf{1}_{W}\left[s_{i, 0}\right]-\mu_{2}\left(\zeta_{i, 0}\right) \zeta_{i, 0}^{\prime}+ \\
& \quad \sum_{j=0}^{K_{i}^{x}(t)}\left[\mu_{h_{i}(j)}\left(\zeta_{i, j+1}\right) \zeta_{i, j+1}^{\prime}-\mu_{h_{i}(j)}\left(\zeta_{i, j}\right) \zeta_{i, j}^{\prime}\right](24)
\end{aligned}
$$

where as earlier, $\zeta_{i, j}^{\prime}$ is the derivative $\zeta_{i, j}$ with respect to $\theta_{W}$. The event time derivatives are determined by Lemmas 1, 5 and 6 .
Theorem 4. The derivative of $Y_{T}(\boldsymbol{\theta})$ with respect to $\theta_{W}$ is given by

$$
\frac{\partial Y_{T}(\boldsymbol{\theta})}{\partial \theta_{W}}=\sum_{i=1}^{N_{T}^{y}} \sum_{j=1}^{K_{i}^{x}+1} y^{\prime}\left(\zeta_{i, j}\right)\left(\zeta_{i, j}-s_{i, 0}\right)
$$

where $y^{\prime}\left(\zeta_{i, j}\right), j=1, \cdots, K_{i}^{x}+1$ is the derivative of $y(t)$ with respect to $\theta_{W}$ and is evaluated by (24) for all $i=1, \cdots, N_{T}^{y}$.

Theorems 1-4 constitute an algorithm for evaluating the sample derivatives of interest with respect to $\theta_{F}$ and $\theta_{W}$. These are similar with the ones derived in Panayiotou et al. (2002) but differ in that they include a number of "correction" terms that account for the fact that the input processes are no longer piecewise constants.

\subsection{Unbiasedness}

Theorem 5. This estimators obtained in Theorems 1-4 are unbiased estimators of the objective functions, i.e.,

$$
\begin{gathered}
\frac{\partial \mathbb{E}\left[X_{T}\right]}{\partial \theta_{F}}=\mathbb{E}\left[\frac{\partial X_{T}}{\partial \theta_{F}}\right], \frac{\partial \mathbb{E}\left[X_{T}\right]}{\partial \theta_{W}}=\mathbb{E}\left[\frac{\partial X_{T}}{\partial \theta_{W}}\right], \\
\frac{\partial \mathbb{E}\left[Y_{T}\right]}{\partial \theta_{F}}=\mathbb{E}\left[\frac{\partial Y_{T}}{\partial \theta_{F}}\right], \frac{\partial \mathbb{E}\left[Y_{T}\right]}{\partial \theta_{W}}=\mathbb{E}\left[\frac{\partial Y_{T}}{\partial \theta_{W}}\right] .
\end{gathered}
$$

\section{REFERENCES}

C. G. Cassandras and S. Lafortune. Introduction to Discrete Event Systems. Kluwer Academic Publishers, 1999.

C. G. Cassandras, Y. Wardi, B. Melamed, G. Sun, and C. G. Panayiotou. Perturbation analysis for on-line control and optimization of stochastic fluid models. IEEE Transactions on Automatic Control, AC-47(8):1234-1248, 2002.

C.G. Panayiotou, C.G. Cassandras, and P. Zhang. On-line inventory cost minimization for maketo-stock manufacturing systems. In Proceedings of the IEEE American Control Conference, pages 4469-4474, 2002.

I. Ch. Paschalidis, Y. Liu, C.G. Cassandras, and C. Panayiotou. Inventory control for supply chains with service level constraints: A synergy between large deviations and perturbation analysis. In Annals of Operations Research, Special Volume on Stochastic Models of ProductionInventory Systems, volume 126, pages 231-258, 2004.

R. Suri and B. R. Fu. On using continuous flow lines for performance estimation of discrete production lines. In Proc. 1991 Winter Simulation Conference, pages 968-977, 1991. 